\title{
Discussion on space-based asteroseismology
}

\author{
led by \\ Annie Baglin \\ Observatoire de Paris, LESIA, UMR 8109, pl. J. Janssen, 92195 Meudon, France
}

Baglin: We know the very near future of space asteroseismology very well and we know how to handle it. We hope that CoRoT will be launched successfully. The next step is to use the data in the most efficient way. Then the Kepler asteroseismology program is to follow, and this is the near future for us.

But after that, it's extremely unclear and even very opaque, so nothing may happen for a long time as Jørgen said. At least from the European side, there seems to be a lack of funding for new important, new-generation space missions dedicated to stellar physics. The idea of imaging stars, which was mostly born in the States, leads certainly into a very bright future. We will probably have to combine all the efforts, the knowledge, and the money of the whole planet to do that. But that's still very far; we have to do something in between. This is an open question that I would like to discuss now.

Certainly MOST has shown the way of how to work on a very cheap level. This is certainly a way to do something from space on a larger basis, to increase the number of projects. The other way is to rely on ground-based observations. Science asks for both space and ground. We have to say everywhere and claim that we need both, that the information we get is different and complementary. It is difficult but it's probably easier to fund a ground based project for at least several years. Even in this discussion, which is mostly dedicated to space, we need to think about what we can do from the ground.

What is your feeling after this meeting, where we made the effort to discuss all these projects that we have in hands and how do you think that we should move on? What strategy should be developed?

Kjeldsen: I think that right now is actually a bad moment to think what we should do next in space. We now have all these nice simulations, but we actually have no data yet except for a few missions like MOST and WIRE. So I think in a few years from now, when we actually have the CoRoT data and the Kepler data, of course we will find that things don't look like the simulations we did and that there will be lots of things that we would have liked to do differently. And that will be the point where we can say what we shall do in the future. I think at the moment it's about analysing the data that the next step is. How are we actually going to use all these many many data? That's where the planning effort should be put at the moment, and not at the next space missions especially because there seems no way to pay for this.

Roxburgh: Part of me agrees with you, but part of me does not because it takes twenty, twenty-five years to get a space mission from concept to launch. We should not stop thinking and agitating. Logically you should first analyse all the data from, e.g., Kepler, by which time it will be 2015 . And then, another twenty years after that, the next mission is due. So, in reality, you need to start planning now. A mission like CoRoT was first thought about in 1981 !

Baglin [to Kjeldsen]: I partly agree with you, but we have to do both. The question is, how much energy do you have to put into each task? But I would like to keep from what you said, that there is a need for a stronger organization to get the best science out of the data we will get and I am not sure that we are ready for that. As you say, it's an enormous amount of data. 
Schrijver: It is a very complex thing that we have to think about what we might be doing twenty years from now even as we actually have to decide now because of the long process ahead. But I would like to stress that this community must think about it rather than letting ESA or NASA separately decide through their committees. You should form a consensus in your community about a few key things you want to do. It's the most important thing to do to get things the way you want. The first step is to decide what's important!

Frandsen: Based on my experience with space projects, where I have seen three failures, starting with PRISMA, then STARS and Eddington, I think we need to think about something different, and Karel's project is maybe the way. Stellar imaging as a solution is really new, and we won't be alone in that area because there are many astronomers who would love to see imaging data. If we can do something that cannot be done from the ground, and we have the possibility to go to the UV, this could be a point to convince the responsible people that this is new and interesting. My advice therefore is to go to something different; interferometry might be such a case.

Kaye: Does anyone know about the optical interferometers that were on range?

Schrijver: Yes, they are still being developed. In terms of imaging of stars, however, they don't have the required baseline and they have to be reconfigured rapidly in order to cover Fourier space. That's why we are asking for something that's really challenging. But people are still working on a ground-based system with something in the range of two to five or six optical elements.

Baglin: VLTI is working. It's not foreseen to do seismology, but there could be some attempts with this kind of array.

Matthews: Picking up on Søren's statement: One of the things that benefited MOST and that helped to get it funded so quickly was that we did not have to co-opt the entire community. We were not competing to take money off other Canadian space astronomers; we got money from a space technology pot that no other astronomer ever thought of using. We needed no peer-review, which was a big factor. One other thing one has to take into account, depending on how expensive the mission is: You have to get everybody in your community for support on board, because it's going to be a major chunk of investment for the community. There are examples of specialized missions at lower budgets in which one can identify a certain science goal, like Werner's demonstration of the BRITE nanosats. There are certain dedicated focused things that one can consider, that may probably get funding without having to have that sort of political support. One should also always keep those in mind to check the skyscape for some more ambitious missions. The community has to decide what the priorities are, but we have to realize that our community is a subset of a much larger community who also needs to buy into that, if we want, say, half a billion Euro to go for.

Roxburgh: If we look at the European mission Eddington, it was more successful than earlier proposals that were not funded. But what also happened at the time was the increasing emphasis on planets. The same is true in recent times. From that I learnt we really ought to be thinking about projects that share with some other areas of astronomy, not just on our own, and something that was equally practicable and wanted by others.

Shipman: What lan just said reminded me of Artie's talk. We can do planets as a byproduct of everything else that we are doing. Artie mentioned that incidentally, but he found planets! So we have at least part of our hat on that. I think we need to think of other things where we can hang our hat on. Just one example: g-modes probe the interior of stars. Why should we want to do that? Well, the size of the core of some stars determines stellar lifetimes, which tells us about stellar populations, which is going to tell us about - a-ha! the evolution of the universe. These kind of things I haven't heard a great deal about here.

Roxburgh: Really, the planets is what has gotten us this far.

Kepler: We have lost the ability to work in the ultraviolet for hot stars. The application of ultraviolet data is much wider in stellar seismology than in other areas, and no one mentioned this possibility here. 
Matthews: I agree with Kepler entirely. The original concept for the Canadian microsat by Slavek Rucinski was for an ultraviolet imaging telescope. It turned out not to be really practical in terms of its mass, etc., and it eventually became MOST. But that led to a project called UVIT and we are now collaborating with the Indians on their ASTROSAT. There are some science drivers here for ultraviolet time-resolved photometry from space of pulsating stars. In fact we are encouraging everyone who is interested to supply proposals and potential targets because this is going ahead.

Kjeldsen: I hope that we really find out with these missions that we don't understand anything inside stars. The worst that can happen is that we confirm everything, that things look like we thought, we measure the ages of stars and basically the theory was OK, because no one will fund another mission doing that. We have to go out and make sure to convince people doing dark matter etc. that stars were not understood in the right way. It changes our view on what physics is, and we may end up in the situation that people are much more willing to support us at the next step. But we have to understand first what these missions really can do. We promised to do asteroseismology but if you look on where we really changed the understanding of the evolution of the universe and so on, we haven't gone where we promised yet, but we hope that space missions can do that. We have to make sure that the whole community can use all these space data. If we make another effort to spread all these data to students around the whole world, they will all learn the way seismologists study stars. Then we will have a much better chance to fund the next mission.

Weiss: I think not every country is in the same situation. Where there is a space agency, there are usually two different budget items. Usually the astronomers don't have direct access to space money and vice versa. It helps if you have a very successful satellite. Then you can go to the other agency and say, we have lots of data, lots of potential science, that the other agency has paid for, and now we need money to work on them. You can do that in countries where you have such a structure; not all have it.

Baglin: I think there are lots of ideas that came out of this discussion. I would suggest that we continue along these lines to develop the future. It is probably best to gather the different ideas of the community, to compare them and to decide what to do. Thank you! 


\section{Other types of pulsators}

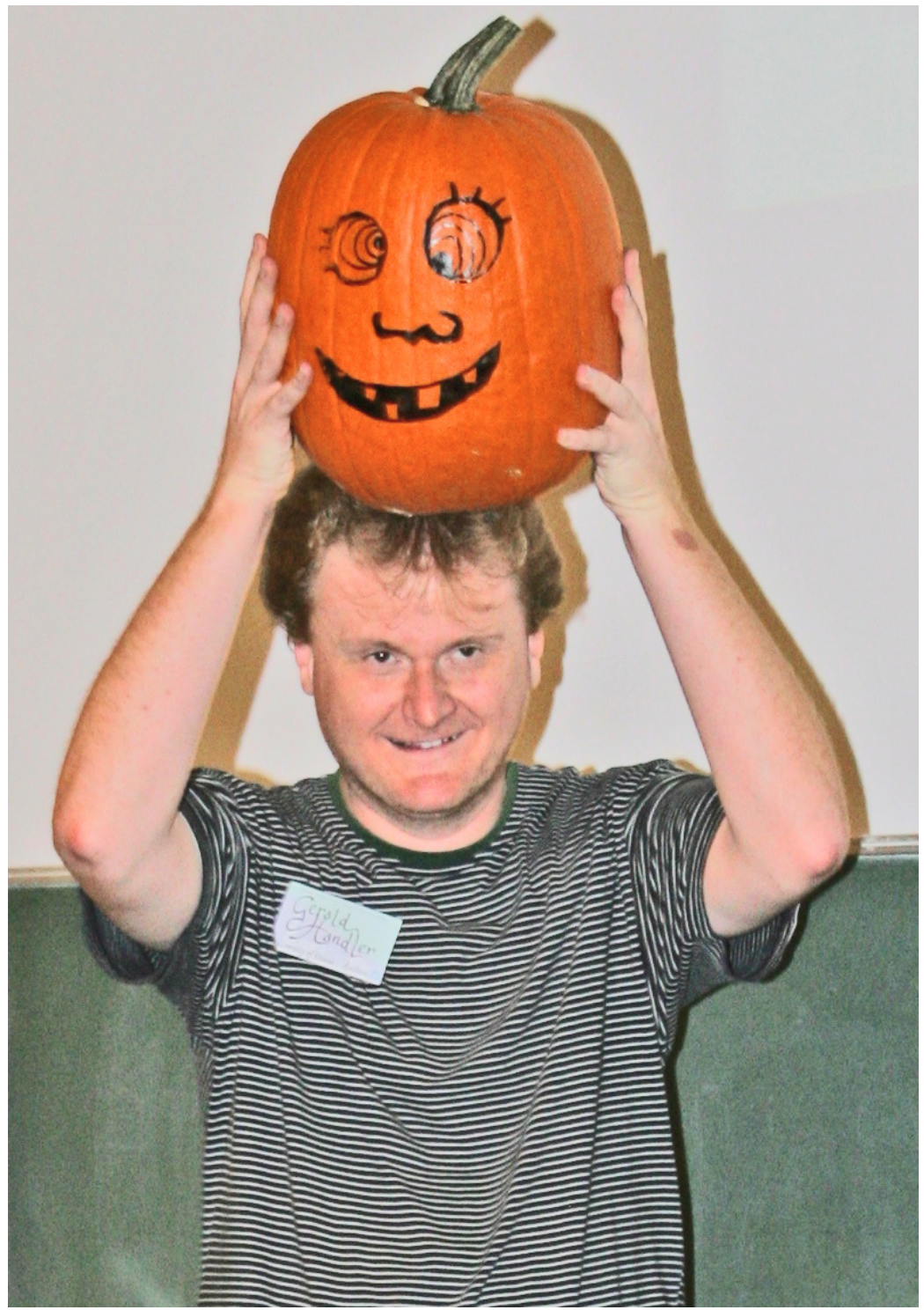

Gerald Handler showing how his head feels at the end of the meeting $(\ell=|m|=16)$. 